\title{
Smart tourism destinations: can the Destination Management Organizations exploit benefits of the ICTs? Evidences from a multiple case study
}

\author{
Salvatore Ammirato ${ }^{1}$, Alberto Michele Felicetti ${ }^{1}$, Marco Della Gala ${ }^{2}$, Cinzia \\ Raso $^{1}$, Marco Cozza ${ }^{3}$ \\ ${ }^{1}$ Department of Mechanical Energy and Management Engineering, University of \\ Calabria, Via P.Bucci, 87036 Rende (CS), Italy \\ \{salvatore.ammirato. alberto.felicetti, cinzia.raso\}@unical.it \\ ${ }^{2}$ Countryside and Community Research Institute, University of Gloucestershire, Oxstalls \\ Campus, Oxstalls Lane GL2 9HW Gloucester, UK \\ mdellagala@glos.ac. \\ 3 3D Research srl, via Bucci, 45\C, 87036 Rende (CS) \\ marco.cozza@3dresearch.it
}

\begin{abstract}
Recent developments of ICTs enable new ways to experience tourism and conducted to the concept of smart tourism. The adoption of cutting-edge technologies and its combination with innovative organizational models fosters cooperation, knowledge sharing, and open innovation among service providers in tourism destination. Moreover, it offers innovative services to visitors. In few words, they become smart tourism destinations. In this paper, we report first results of the SMARTCAL project aimed at conceiving a digital platform assisting Destination Management Organizations (DMOs) in providing smart tourism services. A DMO is the organization charged with managing the tourism offer of a collaborative network, made up of service providers acting in a destination. In this paper, we adopted a multiple case studies approach to analyze five Italian DMOs. Our aims were to investigate 1) if, and how, successful DMOs were able to offer smart tourism services to visitors; 2) if the ICTs adoption level was related to the collaboration level among DMO partners. First results highlighted that use of smart technologies was still in an embryonic stage of development, and it did not depend from collaboration levels.
\end{abstract}

Keywords: Destination Management Organizations, Smart Tourism Services, Multiple case studies 


\section{Introduction}

The World Tourism Organization defined a Tourism Destination (TD) as "a physical space with or without administrative and/or analytical boundaries in which a visitor can spend an overnight. It is the cluster (co-location) of products and services, and of activities and experiences along the tourism value chain and a basic unit of analysis of tourism. A destination incorporates various stakeholders and can network to form larger destinations" [1]. In the same report, the authors stated "to compete effectively, destinations have to deliver wonderful experiences and excellent value to visitors". The business of tourism is complex and fragmented and from the time that visitors arrive in the destination, until they leave it, the quality of their experience is affected by many services and interactions, including a range of public and private services, hospitality services, interactions with communities and environments.

Collaborative Networks represent a real opportunity for tourism operators of a TD to remain competitive in the aggressive global market [2]. With the establishment of a collaborative network in a TD, local tourism operators and tourists can be engaged in social relations and actively participate in continuous experience-based learning processes. Although in a TD live and operate many autonomous and heterogeneous entities, they all aim to achieve local tourism development and to increase their general competitiveness in respect to other TDs as common goal [3] [4]. Destination management calls for a coalition of different interests to work towards a common goal to ensure the viability and integrity of destinations "now, and for the future". A Destination Management Organizations (DMO) is the actor charged with strategic planning, managing, and organizing destination resources. To foster sustainable development practices in a destination, DMOs need to work closely with government agencies, local authorities, businesses, the tourism industry, and other destination stakeholders, to be effective in their role [5].

The role of a DMO should not rely just on marketing and management the destination. DMOs are required to reduce information asymmetries among stakeholders. In this vein, the massive adoption of ICT in many operational contexts related to the tourism experience is helping DMOs to operate as knowledge-based organizations [6].

Recent innovation in ICTs and their interconnections, combined with abilities to infer and reason on big data through artificial intelligence, have had a significant impact on tourism sector [8]. In particular, the sensor technology and the spreading of smartphones and ubiquitous technologies (RFID, NFC, BLE beacon and the Internet of Things - IoT) have enabled the collection, analysis and exchange, of realtime context-aware data, providing insights of digital and physical worlds [8]. In addition, use of web 2.0 and customer reviews on social media, have become important sources of information for both tourists and tourism operators. Tourists 
can use available information for their trip planning, while, service providers can use social media analytics tools for the tourists' digital footprint mining, with a value for their marketing activities [9]. The convergence of these technologies offers the potential to develop information systems able to supply tourists and tourism operators with information that is more relevant, and, in the end, to offer more enjoyable tourism experiences [10]. The adoption of such technologies, combined with the appropriate collaborative business models, support the rise of the smart tourism destinations, i.e. innovative tourist destinations built on an infrastructure of cutting-edge technologies and innovative organizational models, which supports the visitors' interaction with and integration into their surroundings, increasing the quality of their experience [11]. At the same time, smart tourism destinations enhance cooperation, knowledge sharing, and open innovation among service providers [12]. The combination of ICTs with collaborative tourism networks offers noteworthy opportunities to tourism destinations for internal business process re-engineering. It supports the provision of increasingly personalized tourist experiences and help tourism destinations to gain competitive advantage and to adopt sustainable development pathways [13] [7].

This paper reports main results of a multiple case studies research among five Italian successful DMOs. The study aims at investigating if selected DMOs are able to exploit the potentials of ICT. In other words, we investigate on the ability of a small sample of DMOs to exploit ICT potential in supporting the transformation of the respective managed destinations in real smart tourism destinations.

\section{Theoretical Background}

To favor the rise of a smart tourism destination, a DMO needs to become a boundary spanner between a collaborative network of local service providers and tourists. Its main role is to generate and disseminate information and knowledge [5]. A DMO able to reach this aim, lead its destination to become a Smart Tourism Destination. To support this transformation, a DMO has to provide services based on a variety of ICTs to both sides of its boundaries: the front end, meaning the ICTs useful to support the tourist 2.0, and the back end, meaning the set of technologies aimed at supporting the decisional and operational processes within the tourism destination [7][14].

\subsection{Front end: ICT at the tourist 2.0 side}

From a visitor perspective, the availability of Web 2.0 tools and the provision of new personalised informative services can enrich a tourism experience. This is possible thanks to the spread of mobile devices. They enable the ubiquitous access 
to technologies including context-aware systems, augmented realities, autonomous agents searching and mining, ambient intelligence and recommender systems. For example, a tourist might be assisted with attractions, routes and tours recommendations, tourism services recommendations and personalized multiple-days tour planning. To provide these services it could be used a system able to reason and infer on context data. The system could use user location (extracted from GPS receivers, or through Wi-Fi, cell-id, RFID, etc.), time of day, current weather conditions and forecast, user profile information (in some case extracted from social networks), user constraints and preferences, attractions already visited, location and opening hours of POIs, collaborative user-generated content (e.g., comments, attractions ranking, photographs/videos [15]. The above-mentioned technologies can enrich each of the four phases of the so-called tourist 2.0 lifecycle, i.e. the sequence of activities that a tourist usually perform when lives a tourism experience [7]. The phase identified in [7] are dreaming (the emergence of a need, a desire to travel), planning (defining the details of the trip), experiencing (carry-out in-place tourism activities) and recollecting (remembering, memories of the in-place tourism experience).

In particular, for each stage of the lifecycle, we can give further examples related to the use of innovative ICTs. In the dreaming phase, tourists are looking for holiday ideas. At this phase, inspiration portals offer tools for sharing and searching geotagged multimedia contents and reviews, and enable tourists to get a virtual preview of the holiday [16]. In the planning \& booking phase, tourists compose their holiday combining and booking transportations, accommodations, and services (excursions, events, etc.). At this stage, recommender systems and comparison web services allow online users to cope with the information overload [17]. In the experiencing phase, related with in-place tourism activities, context-aware systems, augmented reality and ambient intelligence, combined with the use of RFID, NFC, BLE beacon, sensors, actuators, mobile devices and the IoT, enrich the tourist experience [9]. The integration of these technologies enable the provision of services (maps, location-based services, recommender systems, etc.) affecting the way tourists 2.0 interact with the tourism destination. Recommender systems have become valuable for tourists, especially when they are able to reason and infer on data gathered from the context of the human-sensors interaction to assist users in their decisional processes [18]. They can supply tourists with highly accurate and effective tourist recommendations that capture usage, personal and environmental contextual parameters and respect personal preferences [18]. The recollecting phase is related to the tourist comes back home. Sharing services are used to recall tourist memories on visited places through photos, videos and stories and to give other prospective tourists tips on the experienced tourism destination.

\subsection{Back office: ICT at the DMO side}


From a business perspective, since the mid-nineties, ICTs have had a significant impact in the efficiency and effectiveness of tourism organizations and on their interactions with consumers. Web 2.0 and mobile ICTs support operations, business transaction and networking among partners in the tourism industry [12]. They enable operators to develop original ways to manage the tourism supply chains, the destination marketing and the relations with customers. Technological platform dynamically interconnecting stakeholders and exchanging real-time information on tourism activities, and social media represent important coordination mechanisms. They allow information and knowledge to flow more easily through networked actors operating in a tourism destination and more contextual data to be transmitted [19]. Moreover, the use of AI techniques, in particular artificial neural network (ANN) models, have become an essential tool for economic modelling and forecasting [20]. At the same time, the use of AI, information retrieval and natural language processing, lead to automatic discovery, analysis, and generalisation of tourism consumer views and opinions on tourism destination. Via the automatic recognition of semantic relationships between tourism product features and attributes, and consumer opinions and satisfactions, tourism organizations might control, evaluate and, eventually correct, their marketing strategies [21] [22]. Furthermore, big data analytics techniques for processing, modelling, and visualizing data, gathered during the whole tourist 2.0 life cycle, could generate more detailed information on visitors' spatial and temporal behaviour at the destination. Tourism organizations might exploit these information to formulate planning policy aimed at managing the tourist flows. They could reduce congestions in some areas, and encourage tourists to explore other less visited sites, or to buy less purchased services [23].

All the descripted technologies might improve the success of a tourism destination only in association with the appropriate social structure, and relationships among human actors and organisation in a tourist destination [7]. In particular, as regard tourism operators, as highlighted in [7], they may interact at four level of integration: networking, coordination, cooperation, collaboration; characterized by an incremental amounts of common goal-oriented risk taking, commitment, and resources sharing [24]. At the networking level, DMOs enable the information sharing among tourism service providers, who communicate one another for mutual benefit, but without a common goal. Tourism operators benefit from DMOs' communication and promotional activities, but each operator is responsible for his own services. The most common ICTs are inspiration portals [16], tourism services comparators [25], tourism social networks [17], and mobile and immersive technologies [7]. Moreover, DMOs, when equipped with analytics tools, share data on the market trends and users' perception of a destination to all the networked operators. At the coordination level, it is possible to observe a more 
organizational commitment. Members of the network, even if with different goals and using their own resources, align/alter their activities with the aim to achieve results more efficiently. To satisfy customers' needs, a tourism operator, thanks to the help of the DMO, might "extend" its business services, offering complementary services provided by other tourism operators. In addition to previous technologies, Destination Management Systems - DMSs become important. DMSs support automation of inter-organizational business process collecting into a single portal a variety of services provided by heterogeneous tourism operators located into a specific geographical area [26]. At the cooperation level, tourism operators share knowledge and resources to achieve compatible goals. The combined value is the addition of individual "components" of value generated by the participants in a quasi-independent manner. A DMO provides web-services to compose a customized tourism packages to tourists. At this level, in addition to the previous technologies, Tourism Dynamic Packaging Systems -TDPS are the most suitable solutions. They provide full automation through online applications; real-time update of travel product information; single price for an entire tourism package; guide consumers in the choice of products to add to the package, taking into account the compatibility with products previously added [27]. At collaboration level, all entities share risks, resources, responsibilities, and rewards to achieve a common goal. In this case, the DMO acts as the unique interface of the network. It allows customers to compose a tailored tourism package in a transparent way and it is responsible for its correct provision. At this level the planning and management process, is not only limited to packaging systems but regards many operative and supporting processes which are managed in a common way. In addition to TDPS, Enterprise Resource Planning - ERP technologies for networked tourism organizations represent an important tool to coordinate the network as a whole and to support the accomplishment of shared business processes.

\section{Methodology}

Considering the exploratory nature of this research, we approached it as a multiple case study [28]. According to Fink's suggestion [29] to use a small sample of population members, we selected a sample of convenience of five Italian DMOs, successful from an economic perspective. The focus on the specific country is justified by the leading role played by Italian tourism worldwide [30] [31].

In the scientific literature, there are plenty of studies related to the assessment of determinants of the economic "success" of a DMO. In [32] and [33] exhaustive reviews on empirical studies and models to measure the success of DMOs are reported. The literature review highlights that success of DMOs and of TDs are 
strongly related. In this study, we defined a DMO as successful when it shows positive values on two indexes (the only two indexed on which scholars agree):

- Evolution across time of the number of presences and arrivals in the TD

- Evolution across time of the number of networked partners of the DMO

To individuate the unit of analysis, we analyzed websites of Italian DMOs showing positive values on both indexes in the period 2014-2015. We retrieved website URLs searching specific keywords ("DMO", "destinazione turistica", "località turistica", “...", etc.) on the Google search engine. Information gathered from websites were then validated analyzing articles from newspapers and official papers published by refereed public associations (Italian chambers of commerce, the Italian ministry for Tourism and Cultural Heritage, Confturismo, Federalberghi, regional councilors of tourism, etc.).

Following Yin [28], we collected data by both a careful analysis of documentary sources and by the means of semi-structured interviews. Over the last three months of 2017, two researchers interviewed managers of each DMO. The questions asked during the semi-structured interviews were organized in four blocks: DMO's performances (economic data, visitors, tourist flows, etc.), DMO's members (type of service, number of structures, type of affiliation), DMO's services and ICTs adopted by DMO members.

\section{Results}

\subsection{The cases}

Following Flyvbjerg's [34] suggestions, we choose the following critical case studies among Italian DMOs. We stipulated a "Non-disclosure agreement" with the DMOs we analyzed, whose names cannot be mentioned here without violating the anonymity of the case companies.

$D M O A$ is a limited company providing organizational and administrative services to tourism related companies and it is responsible for the promotion, organization, management and coordination of tourism activities. Moreover, $D M O$ $A$ manages tourism services booking through its web portal. DMO $A$ operates in a municipality that is a renowned winter and summer destination in the Alps, in the northern part of Lombardia Region. DMO's network consist of over 1,700 affiliated facilities (over 110 hotels, 90 restaurants, 2 trade associations, 1 museum, 8 travel agencies and many other suppliers of leisure activities).

$D M O B$ is a public company operating in the tourism promotion of a metropolitan city in central Italy. The city attracts millions of tourists each year and it is famous for its culture, Renaissance art and architecture and monuments. $D M O$ $B$ manages 10 municipal museums and, through its web portal, it promotes tourism services, accommodation, facilities and restaurants. There is no a structured form of affiliation for tourism operators to $D M O B$. However, a loose form of partnership is 
envisaged for the operators who decide to join the service called "Card +", which allows tourists to obtain some benefits (eg. discounts).

$D M O C$ is a public consortium that aim to attract tourists and promoting conference and leisure tourism in a province located in the Veneto Region of Italy. The DMO consists of over 130 hospitality services, 45 restaurants and 4 suppliers of transport services. Furthermore, the DMO comprises 4 companies providing conference facilities, 3 companies specialized in organizing congresses, 2 destination management companies, 2 audio-visual service providers, and 2 providers of conference and interpreting services.

$D M O D$ is responsible for promoting a province in the Piedmont Region as a tourist destination for leisure, sport, nature, culture, individual and group trips, conferences, conventions, incentive travel and business travel. The organizational network $D M O D$ is made up of 3 trade associations, 2 suppliers of leisure activities, 12 incoming tour operators, 3 service providers, 1 hotel consortium, 55 municipalities. Moreover, the DMO gives visibility to all the accommodation facilities through its web portal.

$D M O E$ is a public economic body of an autonomous region located on the northeastern part of Italy. The mission of $D M O E$ is to develop the regional tourism system cooperating with all active tourism operators and supplying them with guidelines to make promotional activities consistent. The DMO's network includes several tourist guide associations and tourism consortia, more than 20 incoming tourism agencies, 7 transport service providers, as well as museums, trade associations, public bodies and recreational activities providers. The DMO web portal gives visibility to all the accommodation facilities in the whole region. Moreover, these facilities can join the online booking service provided by $D M O E$, by paying a fee. To date, about 3,000 tourism services are accessible through the web portal, and about 1.170 of them can be booked thanks the booking service offered by $D M O E$.

The above-described Italian DMOs represent successful DMOs since the two indexes of success agreed in the extant literature are positive for all of them. The following table reports the success indexes for each case study. If compared with the growth rate of the Italian Tourist market in the same period $(+4,4 \%$ evolution of arrivals in 2014-2015), it is noteworthy that all the sampled DMOs present higher values [35]. This reinforces the assertion that the five surveyed DMOs are successful.

Table 1. The success indexes for each case study.

\begin{tabular}{llllll}
\hline Indicators & $D M O A$ & $D M O B$ & $D M O C$ & $D M O D$ & $D M O E$ \\
\hline $\begin{array}{l}\text { Evolution of the } \\
\text { number of } \\
\text { presences (2014- }\end{array}$ & $+9,35 \%$ & $+2,47 \%$ & $+3,50 \%$ & $+8,84 \%$ & $+5,11 \%$ \\
$\begin{array}{l}\text { 2015) }\end{array}$ & & & &
\end{tabular}




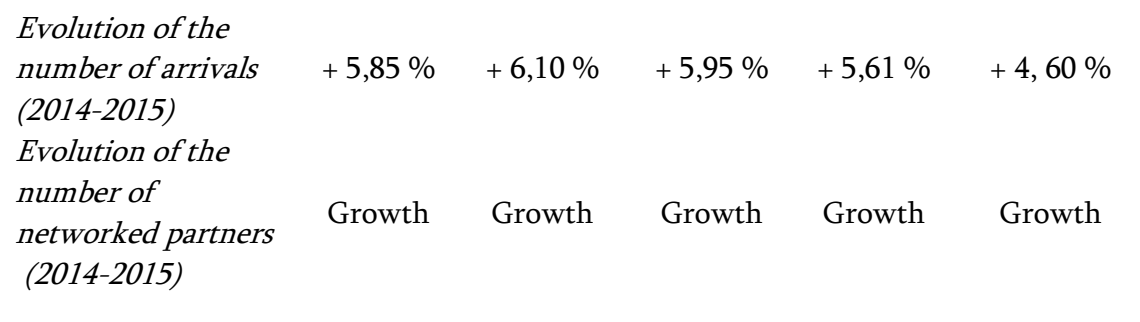

\subsection{Findings}

The analysis of the case studies allowed us to identify ICT solutions adopted by the DMOs to support tourists during the whole tourism experience life cycle and to enable stronger interactions among tourism operators.

With reference to the dreaming phase, it emerges that all the analyzed DMOs use an inspiration portal to promote the destination. It allows tourists to have a preview of the territories, the cultures and the type of holiday they will experience. Moreover, DMOs are equipped with official pages and accounts on the main social networks (Facebook, Pinterest, Twitter, YouTube, Instagram, Google+). DMOs are very active on their social channels and constantly updated their content (in particular $D M O A$ and $D M O E$, are characterized by a high degree of users' involvement). All the DMOs analyzed, except for $D M O C$, propose services they offer on interactive maps accessible through DMOs' web portals. Moreover, they offer content in different languages and include sections containing photo galleries, videos, brochures and multimedia guides.

For what concerns the planning and booking phase, the DMO websites provide sections dedicated to travel planning. DMO $A$ allows tourists to book overnight stays, pre-packaged tourism solutions or customized tourism services. The portal offers the opportunity to make online payments. $D M O C$ and $D M O E$ allow tourists to book tourism services through external links or by filling-in an information request form. $D M O D$ provides tourists with pre-packaged tourism services created by affiliated tour operators. The reservation can be made on $D M O D$ 's web portal. $D M O B$ provides only a "showcase website" allowing tourists to view a list of tourist services in the area. None of the analyzed DMOs proposes the use of advanced and dynamic tools for the creation of tourism packages like TDPS (Tourism Dynamic Packaging System). Although some DMOs adopt ICTs enabling tourists to buy prearranged packages online, only $D M O A$ offers the opportunity to configure tourist packages but it postpones the validation, acceptance and payment phases to be completed offline. Overall, other DMOs portals are configured as an exclusive "static" window of services and products and, therefore, are not able to support tourists in completing in an exhaustive way the planning and booking phase of their tourism experience. 
Analyzed DMOs just barely support the experiencing phase. They do not provide any mobile app to assist tourist. Their websites give tourists the opportunity to obtain information on points of interest, events, tours, public transport, etc., but in the case of $D M O B$ and $D M O C$ the websites are not designed to be responsive, making it difficult for tourists to use them through smartphones, while they are involved in a tourism experience. None of the DMOs offers advanced services such as augmented reality, virtual reality, augmented experience through IoT technologies, guided site exploration, multimedia guides.

During the recollecting phase, the DMOs' websites do not offer a specific section devoted to tourist reviews, stories and memories, except for $D M O D$. Its website presents a specific section where it is possible to leave opinions and evaluations of some tourism products. DMO E examines conversations, comments and reviews on social media in order to detect and improve the "reputation" of the destination. $D M O A, D M O C$ and $D M O D$ use information from online review aggregators to evaluate customer satisfaction.

Overall, the ICTs adoption level for the analyzed DMO is not in line with the latest technological developments. DMOs sufficiently support the dreaming phase of tourist 2.0 lifecycle, while ICT-based services provided during the other phases are very poor. The lack or delay in the adoption of appropriate technologies during the tourist 2.0 lifecycle as a whole does not allow tourists to be involved in a real "augmented tourism experience".

By focusing on ICTs supporting interactions between DMOs and tourism operators, the study shows that $D M O A$ manages operational processes through a DMS (Destination Management System), and institutional communications by using the restricted area of its web portal, social media, e-mail and collaborative working tools. $D M O D$ manages operational processes via e-mail and thanks to a centralized platform with remote access for operators, while it circulates institutional communications by the means of collaborative tools, social media and e-mail. For all other DMOs, there are no significant back-office ICT solutions. Specifically, DMO B manages operational processes by e-mail or telephone, while institutional communications are distributed through the website, e-mails and phone calls; DMO C manages the operational processes by e-mail and telephone contacts, while institutional communications take place via e-mail or external collaborative working tools (for example Google Calendar).

Our analysis highlights that all the DMOs we studied use standard and, sometimes, obsolete tools to support interactions with network operators. They use basic ICTs such as e-mail, social media messaging and telephone contacts to circulate information. For the operational processes management, no DMO uses advanced ICT solutions such as TDPS (Tourism Dynamic Packaging System) or ERP systems (Enterprise Resource Planning). All the studied DMOs are limited in collaborative working tools. 
With reference to the organizational aspects, it is possible to classify each DMO, on the base of its collaboration level as follows: networking for DMO B; coordination for DMO C, DMO D and DMO E; cooperation for DMO A.

Table 2. Adequacy of front-end and back-end technologies.

\begin{tabular}{|c|c|c|c|c|c|}
\hline & $D M O A$ & $D M O B$ & $D M O C$ & $D M O D$ & $D M O E$ \\
\hline Dreaming & (Р) & () & (:) & (ب) & \\
\hline $\begin{array}{l}\text { Planning / } \\
\text { Booking }\end{array}$ & (อ) & & 6 & 6 & \\
\hline Experiencing & & & & & \\
\hline Recollecting & 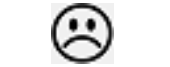 & & & & \\
\hline $\begin{array}{l}\text { Back-end } \\
\text { technologies }\end{array}$ & $\odot$ & & & & \\
\hline $\begin{array}{l}\text { Level of } \\
\text { collaboration }\end{array}$ & $\begin{array}{c}\text { 岸 } \\
\text { cooperation }\end{array}$ & $\underset{\text { networking }}{\text { \# }}$ & coordination & coordination & $\underset{\text { coordination }}{\boxplus}$ \\
\hline
\end{tabular}

\section{Conclusions}

This study was the first of its kind and was based on a multiple case study approach. Since findings of the five cases are not expected to be widely generalizable, they contribute more substantially to the formulation of new hypotheses and to enable subsequent investigations according to other research designs.

In this paper we analyzed how the integration of technologies such as mobile devices, sensors and the IoT, combined with the evolution of artificial intelligence and semantic techniques to infer and reason on big data, have the potential to deeply impact on the tourism sector. We observed how the adoption of these new ICTs by collaborative networks of tourism services providers led by DMOs could bring tourism destination to become "smart", allowing tourists to be better able to enjoy their tourism experience long its whole life cycle. To understand the DMO's ability to offer smart tourism services to destination visitors and to find out any relation between adopted ICTs and the collaboration level among partners in a DMO, we then examined five case studies related to different successful DMOs operating in Italy. Results from the multiple case study show that the overall adoption rate of new technologies in the surveyed DMOs was very low. The five DMOs were using the appropriate ICTs to enhance the dreaming face of the tourism experience life cycle, but they were not supporting the other phase and in particular the 
experiencing one. Moreover, the DMOs we studied were not adopting most advanced technologies to foster the knowledge exchange and collaboration among their partners.

The success in terms of presences and number of networked partners in the 2014-2015 for the studied DMOs might be helped by the driving effect played by Italian tourism worldwide. Anyway, recent studies and analysis on tourist's preferences and needs have been highlighting the shift of the taste of tourists and their needs to search for more authentic and immersive experiences supported by ICTs. To keep continuing to compete and positively perform in the turbulent global market, and possibly to improve their successful performances, it would be worth for the examined DMOs to embrace the new technological development in the sector and to try to exploit the opportunities offered by the adoption of the most recent ICTs. DMOs could thus offer tourists better experiences supporting each phase of the tourism life cycle. At the same time, DMOs are asked to support collaboration among networked tourism service providers offering them systems able to collect, aggregate, analyze, infer and reason on data gathered during the whole tourism lifecycle. Only with such a reasoned approach to ICT adoption, DMOs could be better able to transform the TDs they manage into smart tourism destinations.

Acknowledgments. The research activity reported in this work is funded by MISE (Italian Minister of Economic Development) within the research project "SMARTCAL" (Project number F/050142/02/X32 - CUP B28I15000060008) as part of the "National Operational Programme for Research and Competitiveness 20142020".

\section{References}

1. Fabricius, M., Carter, R., Standford, D.: A practical guide to tourism destination management. World Tourism Organization. (2007)

2. Afsarmanesh, H., Camarinha-Matos, L. M.: Future smart-organizations: a virtual tourism nterprise. In Web Information Systems Engineering, 2000. Proceedings of the First International Conference on, Vol. 1. IEEE (2000) 456-461.

3. Loss, L., Crave, S.: Tourism Breeding Environment: Business Processes Applied to Collaborative Networks in Tourism and Entertainment Sector. In: Camarinha-Matos, L.M., Pereira-Klen, A., Afsarmanesh, H. (eds.) PRO-VE 2011. IFIP AICT, vol. 362, Springer, Heidelberg (2011), 197-204.

4. Ammirato, S., Felicetti, A. M.: Tourism Breeding Environment: forms and levels of collaboration in the tourism sector. In Camarinha-Matos, L.M., Scherer, R. J., (eds.) PRO-VE 2013. IFIP AICT, vol. 408, Springer, Heidelberg (2013), 517-524. 
5. Sheehan, L., Vargas-Sánchez, A., Presenza, A., Abbate, T.: The use of intelligence in tourism destination management: An emerging role for DMOs. nternational Journal of Tourism Research 18(6), 549-557 (2016)

6. Racherla, P., Hu, C., Hyun, M.: Exploring the role of innovative technologies in building a knowledge-based destination. Current issues in tourism 11(5), 407-428 (2008)

7. Ammirato, S., Felicetti, A., Della Gala, M.: Rethinking tourism destinations: collaborative network models for the tourist 2.0. International Journal of KnowledgeBased Development 6(3), 178-201 (2015)

8. Boes, K., Buhalis, D., Inversini, A.: Smart tourism destinations: ecosystems for tourism destination competitiveness. International Journal of Tourism Cities 2(2), 108-124 (2016)

9. Gretzel, U., Werthner, H., Koo, C., Lamsfus, C.: Conceptual foundations for understanding smart tourism ecosystems. Computers in Human Behavior 50, 558-563 (2015)

10. Gretzel, U., Sigala, M., Xiang, Z., Koo, C.: Smart tourism: foundations and developments. Electronic Markets 25(3), 179-188 (2015)

11. Lopez de Avila, A.: Smart destinations: XXI century tourism. In : ENTER2015 conference on information and communication technologies in tourism, Lugano, Switzerland. (2015)

12. Wang, D., Li, X., Li, Y.: China's "smart tourism destination" initiative: A taste of the service-dominant logic. Journal of Destination Marketing \& Management 2(2), 59-61 (2013)

13. Buhalis, D., Law, R.: Progress in information technology and tourism management: 20 years on and 10 year after the Internet - the state of the eTourism research. Tourism Management, 29(4), 609-623 (2008)

14. Ammirato, S., Felicetti, A., Della Gala, M., Aramo-Immonen, H., Jussila, J.: Knowledge Management and Emerging Collaborative Networks in Tourism Business Ecosystems. In : ECKM 2015 - 16th European Conference on Knowledge Management, Udine (Italy) (2015)

15. Gavalas, D., Kenteris, M.: A pervasive web-based recommendation system for mobile tourist guides. Personal and Ubiquitous Computing 15(7), 759-70 (2011)

16. Not, E., Venturini, A.: Supporting Users in Organizing their Vacation Before, During, and After the Travel. In : e-Review of Tourism Research. (2010)

17. Mihajlović, I.: The Impact of Information and Communication Technology (ICT) as a Key Factor of Tourism Development on the Role of Croatian Travel Agencies. nternational Journal of Business and Social Science 3(24), 151-159 (2012)

18. Gavalas, D., Konstantopoulos, C., Mastakas, K., Pantziou, G.: Mobile recommender systems in tourism. Journal of network and computer applications 39, 319-333 (2014)

19. Del Chiappa, G., Baggio, R.: Knowledge transfer in smart tourism destinations: Analyzing the effects of a network structure. Journal of Destination Marketing \& Management 4(3), 145-150 (2015)

20. Claveria, O., Torra, S.: Forecasting tourism demand to Catalonia: Neural networks vs. time series models. Economic Modelling, 36, 220-228 (2014).

21. Akehurst, G.: User generated content: the use of blogs for tourism organisations and tourism consumers. Service business 3(1), 51-61 (2009) 
22. Volpentesta, A. P., Muzzupappa, M., \& Ammirato, S.: Critical thinking and concept design generation in a collaborative network. In Working Conference on Virtual Enterprises. Springer, Boston, MA. (2008) 157-164.

23. Shoval, N.: Tracking technologies and urban analysis., 21-28 (2008)

24. Camarinha-Matos, L. M., Afsarmanesh, H.: Collaborative Networks: Value Creation in a Knowledge Society. In : Knowledge Enterprise: Intelligent Strategies in Product Design, Manufacturing and Management, International Federation for Information Processing (IFIP), Vol. 207. Springer, New York (2006) 26-40

25. Akoumianakis, D.: Ambient affiliates in virtual cross-organizational tourism alliances: A case study of collaborative new product development. Computers in Human Behavior 30, 773-786 (2014)

26. Zanker, M., Fuchs, M., Höpken, W., Tuta, M., Müller, N.: Evaluating Recommender Systems in Tourism - A Case Study from Austria. Information and Communication Technologies in Tourism, 24-34 (2008)

27. Zach, F., Gretzel, U., Fesenmaier, D.: Tourist Activated Networks: Implications for Dynamic Packaging Systems. Tourism Information and Communication Technologies in Tourism, 198-208 (2008)

28. Yin, R.: Case study research and applications: Design and methods. SAGE Publications (2017)

29. Fink, A.: The survey handbook (Vol. 1). Sage (2003)

30. Cuccia, T., Guccio, C., Rizzo, I.: UNESCO sites and performance trend of Italian regional tourism destinations: A two-stage DEA window analysis with spatial interaction. Tourism Economics 23(2), 316-342 (2017)

31. Ammirato, S., Della Gala, M., Volpentesta, A. P.: Alternative agrifood networks as learning communities: some issues for a classification model. In World Summit on Knowledge Society. Springer, Berlin, Heidelberg (2013), 293-300.

32. Bornhorst, T., Ritchie, J., Sheehan, L.: Determinants of tourism success for DMOs \& destinations: An empirical examination of stakeholders' perspectives. Tourism management 31(5), 572-589 (2010)

33. Volgger, M. ., Pechlaner, H.: Requirements for destination management organizations in destination governance: Understanding DMO success. Tourism Management 41, 6475 (2014)

34. Flyvbjerg, B.: Five misunderstandings about case-study research. Qualitative inquiry 12(2), 219-245 (2006)

35. UNTWO Annual Report, accessed on April 2017 available at http://cf.cdn.unwto.org/sites/all/files/pdf/annual_report_2015_lr.pdf (2016) 